\title{
HATS-7b: A HOT SUPER NEPTUNE TRANSITING A QUIET K DWARF STAR*
}

\author{
G. Á. Bakos ${ }^{1,12,13}$, K. Penev ${ }^{1}$, D. Bayliss ${ }^{2,3}$, J. D. Hartman ${ }^{1}$, G. Zhou ${ }^{1,2}$, R. Brahm ${ }^{4,5}$, L. Mancini ${ }^{6}$, M. De VAl-Borro ${ }^{1}$, \\ W. Bhatti ${ }^{1}$, A. Jordán ${ }^{4,5}$, M. Rabus ${ }^{4,6}$, N. Espinoza ${ }^{4,5}$, Z. Csubry ${ }^{1}$, A. W. Howard ${ }^{7}$, B. J. Fulton ${ }^{7,14}$, L. A. BuchHave ${ }^{8,9}$, \\ S. Ciceri ${ }^{6}$, T. Henning ${ }^{6}$, B. Schmidt ${ }^{2}$, H. IsaAcson ${ }^{10}$, R. W. Noyes ${ }^{8}$, G. W. Marcy ${ }^{10}$, V. Suc ${ }^{4}$, A. R. Howe ${ }^{1}$, A. S. Burrows ${ }^{1}$, \\ J. LÁZÁR ${ }^{11}$, I. PAPP ${ }^{11}$, AND P. SÁRI ${ }^{11}$ \\ ${ }^{1}$ Department of Astrophysical Sciences, Princeton University, NJ 08544, USA \\ ${ }^{2}$ Research School of Astronomy and Astrophysics, Australian National University, Canberra, ACT 2611, Australia \\ ${ }_{3}^{3}$ Observatoire Astronomique de l'Université de Genève, $51 \mathrm{ch}$. des Maillettes, 1290 Versoix, Switzerland \\ ${ }^{4}$ Instituto de Astrofísica, Facultad de Física, Pontificia Universidad Católica de Chile, Av. Vicuña Mackenna 4860, 7820436 Macul, Santiago, Chile \\ 5 Millennium Institute of Astrophysics, Av. Vicuña Mackenna 4860, 7820436 Macul, Santiago, Chile \\ ${ }^{6}$ Max Planck Institute for Astronomy, Heidelberg, Germany \\ ${ }^{7}$ Institute for Astronomy, University of Hawaii at Manoa, Honolulu, HI, USA \\ ${ }^{8}$ Harvard-Smithsonian Center for Astrophysics, Cambridge, MA 02138, USA \\ ${ }^{9}$ Centre for Star and Planet Formation, Natural History Museum, University of Copenhagen, Denmark \\ ${ }^{10}$ Department of Astronomy, University of California, Berkeley, CA 94720-3411, USA \\ ${ }^{11}$ Hungarian Astronomical Association, Budapest, Hungary \\ Received 2015 July 3; accepted 2015 September 14; published 2015 November 4
}

\begin{abstract}
We report the discovery by the HATSouth network of HATS-7b, a transiting Super-Neptune with a mass of 0.120 $\pm 0.012 M_{\mathrm{J}}$, a radius of $0.563_{-0.034}^{+0.046} R_{\mathrm{J}}$, and an orbital period of 3.1853 days. The host star is a moderately bright $\left(V=13.340 \pm 0.010 \mathrm{mag}, K_{S}=10.976 \pm 0.026 \mathrm{mag}\right) \mathrm{K}$ dwarf star with a mass of $0.849 \pm 0.027 M_{\odot}$, a radius of $0.815_{-0.035}^{+0.049} R_{\odot}$, and a metallicity of $[\mathrm{Fe} / \mathrm{H}]=+0.250 \pm 0.080$. The star is photometrically quiet to within the precision of the HATSouth measurements, has low RV jitter, and shows no evidence for chromospheric activity in its spectrum. HATS-7b is the second smallest radius planet discovered by a wide-field ground-based transit survey, and one of only a handful of Neptune-size planets with mass and radius determined to $10 \%$ precision. Theoretical modeling of HATS-7b yields a hydrogen-helium fraction of $18 \pm 4 \%$ (rock-iron core and $\mathrm{H}_{2}-\mathrm{He}$ envelope), or $9 \pm 4 \%$ (ice core and $\mathrm{H}_{2}-\mathrm{He}$ envelope), i.e., it has a composition broadly similar to that of Uranus and Neptune, and very different from that of Saturn, which has $75 \%$ of its mass in $\mathrm{H}_{2}-\mathrm{He}$. Based on a sample of transiting exoplanets with accurately $(<20 \%)$ determined parameters, we establish approximate powerlaw relations for the envelopes of the mass-density distribution of exoplanets. HATS-7b, which, together with the recently discovered HATS-8b, is one of the first two transiting super-Neptunes discovered in the Southern sky, is a prime target for additional follow-up observations with Southern hemisphere facilities to characterize the atmospheres of Super-Neptunes (which we define as objects with mass greater than that of Neptune, and smaller than halfway between that of Neptune and Saturn, i.e., $0.054 M_{\mathrm{J}}<M_{\mathrm{p}}<0.18 M_{\mathrm{J}}$ ).
\end{abstract}

Key words: stars: individual (HATS-7) - techniques: photometric - techniques: radial velocities - techniques: spectroscopic

Supporting material: machine-readable table

\section{INTRODUCTION}

The HATSouth project (Bakos et al. 2013) is the first global network of homogeneous, fully automated telescopes, capable of round-the-clock monitoring of a wide field of view (FOV) on the sky.

The key scientific goal of HATSouth is to search for transiting extrasolar planets, especially ones that are smaller

\footnotetext{
* The HATSouth network is operated by a collaboration consisting of Princeton University (PU), the Max Planck Institute für Astronomie (MPIA), the Australian National University (ANU), and the Pontificia Universidad Católica de Chile (PUC). The station at Las Campanas Observatory (LCO) of the Carnegie Institute is operated by PU in conjunction with PUC, the station at the High Energy Spectroscopic Survey (H.E.S.S.) site is operated in conjunction with MPIA, and the station at Siding Spring Observatory (SSO) is operated jointly with ANU. This paper includes data gathered with the $10 \mathrm{~m}$ Keck-I telescope at Mauna Kea, the MPG $2.2 \mathrm{~m}$ and ESO $3.6 \mathrm{~m}$ telescopes at the ESO Observatory in La Silla. This paper uses observations obtained with facilities of the Las Cumbres Observatory Global Telescope.

12 Alfred P. Sloan Research Fellow.

13 Packard Fellow.

14 NSF Graduate Research Fellow.
}

and/or have longer periods than hot Jupiters and Saturns, which have been the primary type of planet found by groundbased surveys to date. By covering a wider total FOV than the NASA Kepler mission (Borucki et al. 2010), HATSouth monitors more bright stars per unit time than this mission, promising a greater yield of planets amenable to detailed characterization.

The HATSouth telescopes are installed at Las Campanas Observatory (LCO) in Chile, at the H.E.S.S. site in Namibia, and at Siding Spring Observatory (SSO) in Australia. HATSouth was commissioned in late 2009, with regular operations beginning in 2011 after a $\sim 1$ year shakedown period, and has since collected 2.9 million science images at 4 minute cadence for some 9.5 million stars with $r<16$ mag ( $2 \%$ per-point precision) in the Southern sky. As of 2015 May 1, the HATSouth telescopes have opened on 1788, 1528, and 1324 nights from LCO, HESS, and SSO, respectively. Based on weather statistics through 2015, the sites have averaged $8.24 \mathrm{hr}, 7.54 \mathrm{hr}$, and $5.24 \mathrm{hr}$ of useful dark hours per $24 \mathrm{hr}$ time period, respectively. The longitudinal distribution of the three 
Table 1

Summary of Photometric Observations of HATS-7

\begin{tabular}{|c|c|c|c|c|c|}
\hline Facility/Field ${ }^{\mathrm{a}}$ & Date Range & Number of Points & $\begin{array}{r}\text { Median Cadence } \\
\text { (s) }\end{array}$ & Filter & $\begin{array}{r}\text { Precision }^{\mathrm{b}} \\
\text { (mmag) }\end{array}$ \\
\hline HS-2/G568 & 2011 Mar-Aug & 5018 & 290 & Sloan $r$ & 7.1 \\
\hline HS-4/G568 & 2011 Jul-Aug & 839 & 301 & Sloan $r$ & 6.9 \\
\hline LCOGT1m+SBIG & 2014 Jun 12 & 110 & 136 & Sloan $i^{\prime}$ & 2.0 \\
\hline LCOGT1m+sinistro & 2014 Jun 16 & 49 & 167 & Sloan $i^{\prime}$ & 1.3 \\
\hline GROND & 2014 Jul 20 & 83 & 140 & Sloan $g$ & 1.2 \\
\hline GROND & 2014 Jul 20 & 80 & 140 & Sloan $z$ & 1.0 \\
\hline
\end{tabular}

Notes.

${ }^{\text {a }}$ For the HATSouth observations we list the HATSouth (HS) instrument used to perform the observations and the pointing on the sky. HS-2 is located at Las Campanas Observatory in Chile, HS-4 at the H.E.S.S. gamma-ray telescope site in Namibia, and HS-6 at Siding Spring Observatory in Australia. Field G568 is one of 838 discrete pointings used to tile the sky for the HATNet and HATSouth projects. This particular field is centered at R.A. $14 \mathrm{hr}$ and decl. $-22.5^{\circ}$.

b The rms scatter of the residuals from our best fit transit model for each light curve at the cadence indicated in the table.

sites means that selected regions of the sky may be observed for long contiguous stretches of time, occasionally reaching $130 \mathrm{hr}$ with interruptions shorter than 30 minutes.

Here we present the discovery of HATS-7b, which together with the recently announced HATS-8b (Bayliss et al. 2015), is one of the first two transiting Super-Neptunes found by the HATSouth network, and one of only four such planets discovered by a ground-based wide-field survey (the other two being HAT-P-11b, Bakos et al. 2010, and HAT-P-26b, Hartman et al. 2011a). By combining photometric observations with high-resolution, high velocity-precision spectroscopy, we determine the mass and radius of HATS-7b to better than $10 \%$ accuracy. HATS-7b is one of only nine planets with $M<0.18$ $M_{\mathrm{J}}$ for which both the mass and radius have been determined to this level of accuracy. ${ }^{15}$ With $V=13.340 \pm 0.010 \mathrm{mag}$, HATS-7 is the 7th brightest star known to host a transiting planet with $M<0.18 M_{\mathrm{J}}$, and for which the mass has been accurately (with $10 \%$ accuracy) determined. And of these planets, only GJ 436 (Gillon et al. 2007), GJ 3470 (Bonfils et al. 2012), and HAT-P-26 (Hartman et al. 2011a) have deeper transits, making HATS-7b one of the best known low-mass transiting planets for detailed follow-up studies, e.g., transmission spectroscopy for probing its atmospheric composition.

In the following section we describe the observations utilized to discover and characterize HATS-7b. In Section 3 we discuss our analysis of the data to rule out blend scenarios and determine the system parameters. We discuss the results in Section 4

\section{OBSERVATIONS}

\subsection{Photometry}

\subsubsection{Photometric Detection}

The star HATS-7 (2MASS 13552567-2112276) was observed by the HATSouth wide-field telescope network between UT 2011 March 24 and UT 2011 August 19. These observations are summarized in Table 1. Observations were made from LCO in Chile, the H.E.S.S. site in Namibia, and

\footnotetext{
15 Based on the NASA exoplanet archive http://exoplanetarchive. ipac.caltech.edu accessed 2015 March 25.
}
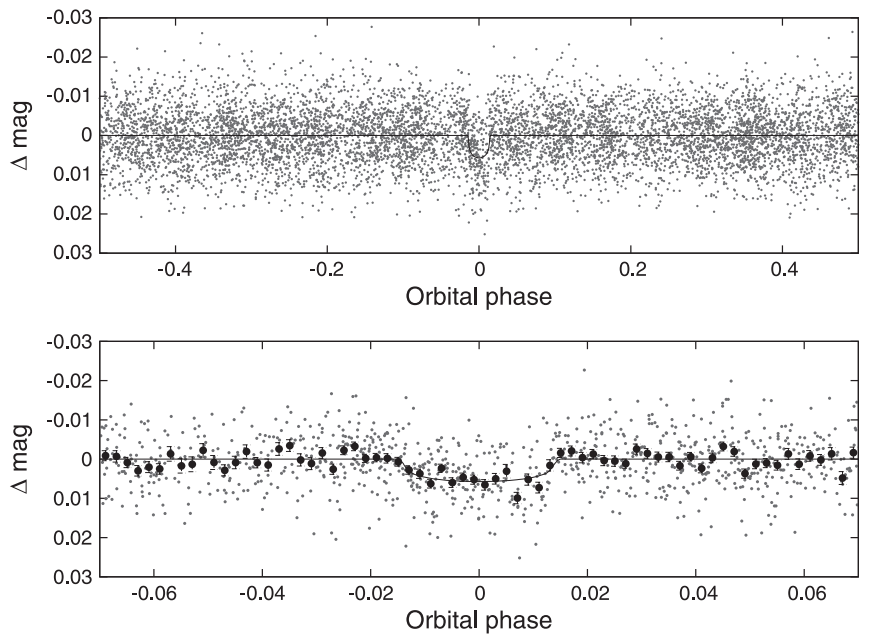

Figure 1. Unbinned instrumental $r$-band light curve of HATS-7 folded with the period $P=3.1853150$ days resulting from the global fit described in Section 3. The solid line shows the best-fit transit model (see Section 3). In the lower panel we zoom-in on the transit; the dark filled points here show the light curve binned in phase using a bin size of 0.002 .

SSO in Australia. The data were reduced to trend-filtered light curves following Penev et al. (2013) and making use of the Trend Filtering Algorithm (TFA; Kovács et al. 2005). We searched the light curves extracted from the images for periodic box-shaped transits using the Box Least Squares (Kovács et al. 2002) algorithm, and identified a $P=3.1853$ day signal with a depth of $5.1 \mathrm{mmag}$ in the light curve of HATS-7 (Figure 1; the data are available in Table 2).

We searched the HATSouth light curve of HATS-7 for other periodic transit signals which would indicate the presence of additional transiting planets in the system, but found no evidence for any such signals. We also searched for continuous periodic variability, due for example to the presence of starspots on HATS-7. No significant periodic signals are present in the TFA-processed light curve, and we place an upper limit of $1 \mathrm{mmag}$ on the peak-to-peak amplitude of any such variability. We also checked for continuous periodic variability in the light curve before applying the TFA filtering, and in this case find that the maximum signal present $(P=39$ days $)$ has a peak-to- 
Table 2

Differential Photometry of HATS-7

\begin{tabular}{lrcccc}
\hline \hline $\begin{array}{l}\text { BJD } \\
(2400000+)\end{array}$ & \multicolumn{1}{c}{ Mag $^{\mathrm{a}}$} & $\sigma_{\text {Mag }}$ & $\left.\operatorname{Mag}_{(\mathrm{orig})}\right)^{\mathrm{b}}$ & Filter & Instrument \\
\hline 55784.52625 & -0.00947 & 0.00553 & $\ldots$ & $r$ & HS \\
55685.78275 & 0.00387 & 0.00490 & $\ldots$ & $r$ & HS \\
55682.59768 & 0.00210 & 0.00476 & $\ldots$ & $r$ & HS \\
55666.67146 & 0.00587 & 0.00552 & $\ldots$ & $r$ & HS \\
55762.23228 & -0.00517 & 0.00421 & $\ldots$ & $r$ & HS \\
55784.52962 & 0.01251 & 0.00600 & $\ldots$ & $r$ & HS \\
55650.74655 & 0.01291 & 0.00467 & $\ldots$ & $r$ & HS \\
55749.49145 & 0.00899 & 0.00508 & $\ldots$ & $r$ & HS \\
55685.78731 & 0.00285 & 0.00494 & $\ldots$ & $r$ & HS \\
55682.60232 & -0.00122 & 0.00467 & $\ldots$ & $r$ & HS \\
\hline
\end{tabular}

Notes. The data are also available on the HATSouth website at http://www. hatsouth.org.

${ }^{a}$ The out-of-transit level has been subtracted. For the HATSouth light curve (rows with "HS" in the Instrument column), these magnitudes have been detrended using the EPD and TFA procedures prior to fitting a transit model to the light curve. Primarily as a result of this detrending, but also due to blending from neighbors, the apparent HATSouth transit depth is somewhat shallower than that of the true depth in the Sloan $r$ filter (the apparent depth is $85 \%$ that of the true depth). For the follow-up light curves (rows with an Instrument other than "HS") these magnitudes have been detrended with the EPD procedure, carried out simultaneously with the transit fit (the transit shape is preserved in this process).

${ }^{b}$ Raw magnitude values without application of the EPD procedure. This is only reported for the follow-up light curves.

(This table is available in its entirety in machine-readable form.)

peak amplitude of $2.7 \mathrm{mmag}$, and is consistent with the level of low-frequency systematic noise present in the pre-TFA light curves of other bright stars in the field of HATS-7.

\subsubsection{Photometric Follow-up}

Photometric follow-up observations of HATS-7 were performed using the 1-m telescopes in the Las Cumbres Observatory Global Telescope network (LCOGT) and the GROND instrument on the MPG $2.2 \mathrm{~m}$ telescope at La Silla Observatory (LSO) in Chile. These observations are summarized in Table 1. Table 2 provides the light curve data, while the light curves are compared to our best-fit model in Figure 2.

Two transits were observed using the LCOGT $1 \mathrm{~m}$ network (Brown et al. 2013). The first, on UT 2014 June 12, was observed using a $4 \mathrm{~K} \times 4 \mathrm{~K}$ SBIG STX-16803 camera, with 0 .' 23 pixels, at the South African Astronomical Observatory station. The second transit, on UT 2014 June 16, was observed using the Sinistro camera at Cerro Tololo Inter-American Observatory station in Chile, which utilizes a $4 \mathrm{~K} \times 4 \mathrm{~K}$ Fairchild CCD-486 back-side illuminated detector with 0." 39 pixels. For both transits we used a Sloan $i^{\prime}$ filter. Because no apparent stellar neighbor is visible on archival images, or through our own observations, we defocused the telescope to reduce systematic errors in the photometry. Standard CCD calibrations were performed and light curves were extracted using standard aperture photometry routines.

A single transit was observed on UT 2014 July 20 using the GROND multi-filter instrument, providing simultaneous observations in the Sloan $g, r, i$, and $z$ band-passes (Greiner et al. 2008). The data were reduced to light curves following Penev et al. (2013) and Mohler-Fischer et al. (2013).

\subsection{Spectroscopy}

Spectroscopic follow-up observations of HATS-7 were carried out with WiFeS on the ANU $2.3 \mathrm{~m}$ telescope at SSO (Dopita et al. 2007), FEROS on the MPG $2.2 \mathrm{~m}$ (Kaufer \& Pasquini 1998), CORALIE on the Euler $1.2 \mathrm{~m}$ at LSO (Queloz et al. 2001), and HIRES on the Keck-I 10 m telescope at Mauna Kea Observatory in Hawaii (Vogt et al. 1994).

The WiFeS observations were carried out as part of our reconnaissance to rule out false positives, and reduced and analyzed following Bayliss et al. (2013) and Zhou et al. (2014). We obtained three observations at a resolution of $R \equiv \lambda / \Delta \lambda=7000$ to check for radial velocity variations in excess of $5 \mathrm{~km} \mathrm{~s}^{-1}$, and found that the observations were consistent with no variation at this level. A fourth spectrum at a resolution of $R=3000$ was obtained to provide an initial spectral classification and estimate of the surface gravity. Based on this observation we found that HATS-7 is a K dwarf star with an estimated effective temperature of $T_{\text {eff } \star}=4700 \pm 300$ $\mathrm{K}$ and a surface gravity of $\log g_{\star}=4.4 \pm 0.3$.

We obtained three $R=48,000$ resolution FEROS spectra and five $R=60,000$ resolution CORALIE spectra, which were reduced and analyzed following Jordán et al. (2014). The RVs measured from these spectra had a rms scatter of $20 \mathrm{~m} \mathrm{~s}^{-1}$ and were consistent with no variation. Based on these observations we concluded that HATS-7b is a probable Neptune-mass planet, and continued observing it with the larger aperture Keck-I telescope. The stellar atmospheric parameters estimated from the FEROS and CORALIE spectra are consistent with those measured from WiFeS. We also determined that the star is slowly rotating (with $v \sin i<5 \mathrm{~km} \mathrm{~s}^{-1}$ ), and, based on inspecting the cross-correlation functions (CCFs), found no evidence for additional stellar components in the spectra.

We observed HATS-7 with the HIRES spectrometer (Vogt et al. 1994) on the $10 \mathrm{~m}$ Keck-1 telescope using standard practices of the California Planet Survey (Howard et al. 2010). Our 10 observations through a gas cell of molecular iodine $\left(\mathrm{I}_{2}\right)$ lasted 25 minutes each using the "C2" decker (14 times 0.86 arcsec slit). In addition, we observed HATS-7 without the $\mathrm{I}_{2}$ cell using the "B3" decker (14 times 0.57 arcsec slit) to record a template spectrum for the RV analysis and for measuring high-precision stellar atmospheric parameters. We computed relative RVs using the Butler et al. (1996) method to model the $\mathrm{I}_{2} \times$ star spectrum. RV errors were estimated from the uncertainty on the mean of 700 spectral segments (each spanning $\sim 2 \AA$ ) that were separately analyzed for each observation. We also measured spectral line bisector spans (BSs) from these data following Torres et al. (2007). The final RVs and BSs are provided in Table 3 and are displayed in Figure 3. The RVs vary in phase with the photometric ephemeris and with a semiamplitude of $K=18.4 \pm 1.9 \mathrm{~m} \mathrm{~s}^{-1}$, while the BSs are consistent with no variation and have an rms scatter of $8.4 \mathrm{~m} \mathrm{~s}^{-1}$.

Based on the Keck/HIRES spectra, HATS-7 is a quiet $\mathrm{K}$ dwarf, with barely detectable Calcium HK activity $\left(\langle S\rangle=0.27, \log R_{\mathrm{HK}}^{\prime}=-4.800\right.$; Table 4). Also, HATS7 has no significant RV jitter (Table 5). It is similar to other very low activity $\mathrm{K}$ dwarfs, like HAT-P-26 (Hartman et al. 2011a).

\section{ANALYSIS}

We analyzed the photometric and spectroscopic observations of HATS-7 to determine the parameters of the system using the 


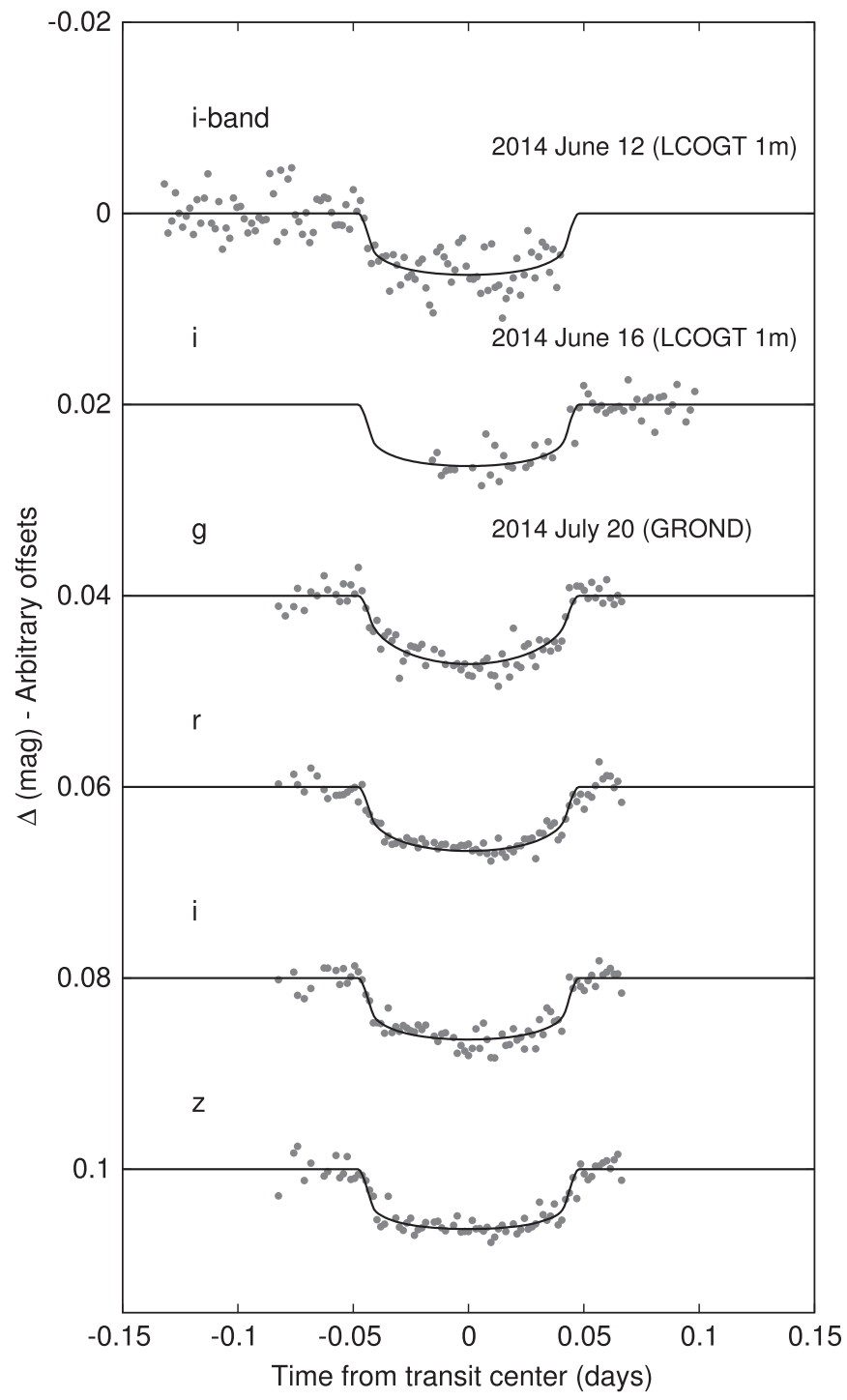

Figure 2. Left: unbinned follow-up transit light curves of HATS-7. The dates, filters and instruments used for each event are indicated. The light curves have been detrended using the EPD process. Curves after the first are shifted for clarity. Our best fit is shown by the solid lines.

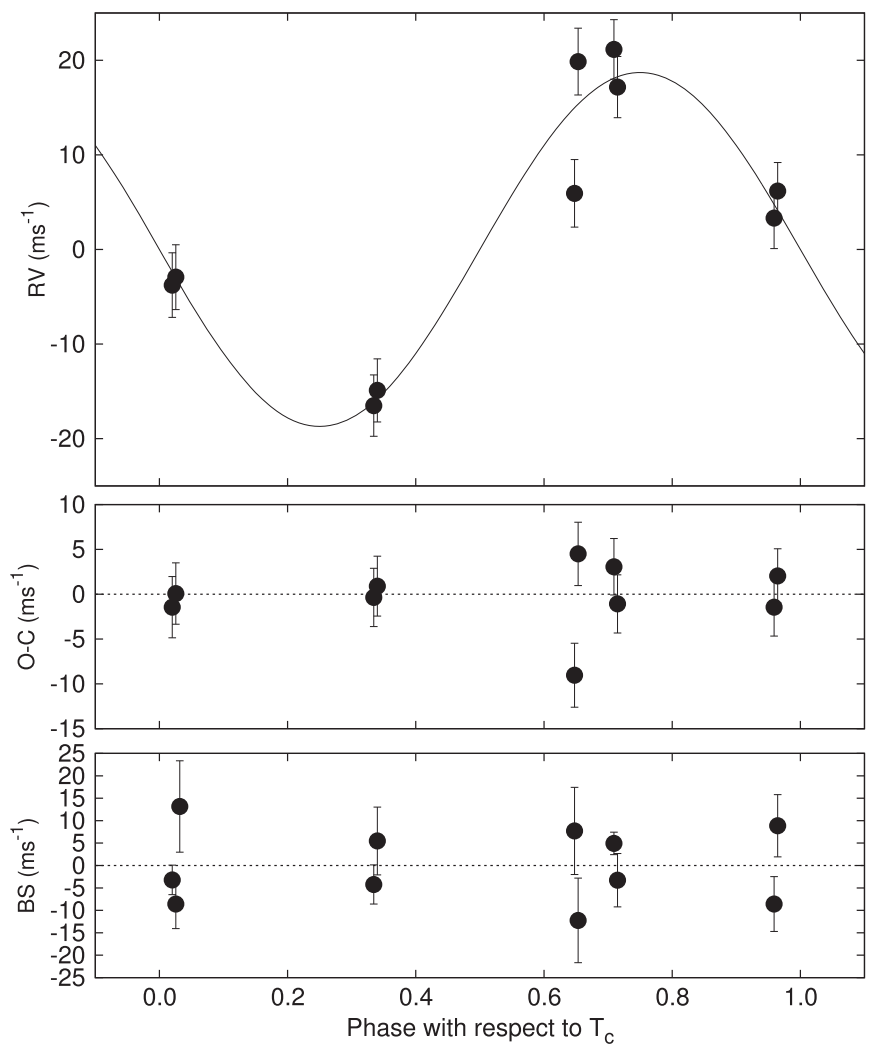

Figure 3. Top panel: high-precision RV measurements for HATS-7 from Keck/HIRES, together with our best-fit circular orbit model. Zero phase corresponds to the time of mid-transit. The center-of-mass velocity has been subtracted. Second panel: velocity $O-C$ residuals from the best-fit model. The error bars for each instrument include the jitter which is determined in the fit. Third panel: bisector spans (BS), with the mean value subtracted. Note the different vertical scales of the panels.

standard procedures developed for HATNet and HATSouth (see Bakos et al. 2010, with modifications described by Hartman et al. 2012).

High-precision stellar atmospheric parameters were measured from the Keck/HIRES template spectrum using the Spectral Parameter Classification program (SPC; Buchhave

Table 3

Relative Radial Velocities and Bisector Span Measurements of HATS-7

\begin{tabular}{|c|c|c|c|c|c|c|}
\hline $\begin{array}{l}\text { BJD } \\
(2456000+)\end{array}$ & $\begin{array}{r}\mathrm{RV}^{\mathrm{a}} \\
\left(\mathrm{m} \mathrm{s}^{-1}\right)\end{array}$ & $\begin{array}{c}\sigma_{\mathrm{RV}}{ }^{\mathrm{b}} \\
\left(\mathrm{m} \mathrm{s}^{-1}\right)\end{array}$ & $\begin{array}{c}\mathrm{BS} \\
\left(\mathrm{m} \mathrm{s}^{-1}\right)\end{array}$ & $\sigma_{\mathrm{BS}}$ & Phase & Instrument \\
\hline 826.78970 & 21.15 & 2.07 & 4.9 & 2.5 & 0.709 & Keck \\
\hline 826.80787 & 17.17 & 2.21 & -3.3 & 6.0 & 0.715 & Keck \\
\hline 827.78004 & -3.77 & 2.46 & -3.2 & 3.3 & 0.020 & Keck \\
\hline 827.79805 & -2.93 & 2.47 & -8.6 & 5.5 & 0.026 & Keck \\
\hline $827.81761^{\mathrm{c}}$ & $\ldots$ & $\ldots$ & 13.2 & 10.2 & 0.032 & Keck \\
\hline 828.78151 & -16.51 & 2.22 & -4.2 & 4.4 & 0.334 & Keck \\
\hline 828.79940 & -14.90 & 2.35 & 5.5 & 7.5 & 0.340 & Keck \\
\hline 829.77933 & 5.93 & 2.67 & 7.7 & 9.7 & 0.648 & Keck \\
\hline 829.79741 & 19.86 & 2.62 & -12.2 & 9.4 & 0.653 & Keck \\
\hline 830.77141 & 3.32 & 2.19 & -8.6 & 6.1 & 0.959 & Keck \\
\hline 830.78899 & 6.17 & 1.88 & 8.9 & 6.9 & 0.965 & Keck \\
\hline
\end{tabular}

Notes.

${ }^{\text {a }}$ The zero-point of these velocities is arbitrary. An overall offset $\gamma_{\text {rel }}$ is fitted to the Keck/HIRES velocities (Section 3), and has been subtracted.

${ }^{\mathrm{b}}$ Internal errors excluding the component of astrophysical/instrumental jitter considered in Section 3.

${ }^{c}$ This HIRES observation was taken without the iodine cell to be used as a template. The RV is not measured for this observations, but the BS value is measured. 
Table 4

Stellar Parameters for HATS-7

\begin{tabular}{|c|c|c|}
\hline Parameter & Value & Source \\
\hline \multicolumn{3}{|l|}{ Identifying information } \\
\hline R.A. (h:m:s) & $13^{\mathrm{h}} 55^{\mathrm{m}} 25.68 \mathrm{~s}$ & 2MASS \\
\hline decl. (d:m:s) & $-21^{\circ} 12^{\prime} 27 ! \prime 7$ & 2MASS \\
\hline 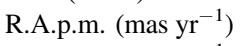 & $-43.3 \pm 1.4$ & 2MASS \\
\hline decl.p.m. (mas $\left.\mathrm{yr}^{-1}\right)$ & $-37.2 \pm 1.9$ & 2MASS \\
\hline GSC ID & GSC 6148-00422 & GSC \\
\hline 2MASS ID & $13552567-2112276$ & 2MASS \\
\hline \multicolumn{3}{|c|}{ Spectroscopic properties } \\
\hline$T_{\text {eff } \star}(\mathrm{K})$ & $4985 \pm 50$ & $\mathrm{SPC}^{\mathrm{a}}$ \\
\hline Spectral type & $\mathrm{K} 2$ & SPC \\
\hline$[\mathrm{Fe} / \mathrm{H}]$ & $+0.250 \pm 0.080$ & SPC \\
\hline$v \sin i\left(\mathrm{~km} \mathrm{~s}^{-1}\right)$ & $0.50 \pm 0.50$ & SPC \\
\hline$\gamma_{\mathrm{RV}}\left(\mathrm{km} \mathrm{s}^{-1}\right)$ & $9.435 \pm 0.017$ & FEROS \\
\hline$\langle S\rangle$ & 0.27 & Keck/HIRES \\
\hline $\log R_{\mathrm{HK}}^{\prime}$ & -4.800 & Keck/HIRES \\
\hline \multicolumn{3}{|l|}{ Photometric properties } \\
\hline$B(\mathrm{mag})$ & $14.359 \pm 0.020$ & APASS \\
\hline$V(\mathrm{mag})$ & $13.340 \pm 0.010$ & APASS \\
\hline$g(\mathrm{mag})$ & $13.825 \pm 0.010$ & APASS \\
\hline$r$ (mag) & $13.013 \pm 0.030$ & APASS \\
\hline$i$ (mag) & $12.690 \pm 0.030$ & APASS \\
\hline$J(\mathrm{mag})$ & $11.528 \pm 0.024$ & 2MASS \\
\hline$H$ (mag) & $11.085 \pm 0.024$ & 2MASS \\
\hline$K_{s}(\mathrm{mag})$ & $10.976 \pm 0.026$ & 2MASS \\
\hline \multicolumn{3}{|l|}{ Derived properties } \\
\hline$M_{\star}\left(M_{\odot}\right)$ & $0.849 \pm 0.027$ & $\mathrm{Y}^{2}+\rho_{\star}+\mathrm{SPC}^{\mathrm{b}}$ \\
\hline$R_{\star}\left(R_{\odot}\right)$ & $0.815_{-0.035}^{+0.049}$ & $\mathrm{Y}^{2}+\rho_{\star}+\mathrm{SPC}$ \\
\hline$\rho_{\star}(\mathrm{cgs})$ & $2.22 \pm 0.33$ & $\mathrm{Y}^{2}+\rho_{\star}+\mathrm{SPC}$ \\
\hline $\log g_{\star}(\mathrm{cgs})$ & $4.545 \pm 0.049$ & $\mathrm{Y}^{2}+\rho_{\star}+\mathrm{SPC}$ \\
\hline$L_{\star}\left(L_{\odot}\right)$ & $0.368_{-0.038}^{+0.050}$ & $\mathrm{Y}^{2}+\rho_{\star}+\mathrm{SPC}$ \\
\hline$M_{V}(\mathrm{mag})$ & $6.10 \pm 0.14$ & $\mathrm{Y}^{2}+\rho_{\star}+\mathrm{SPC}$ \\
\hline$M_{K}(\mathrm{mag}, \mathrm{ESO})$ & $3.94 \pm 0.11$ & $\mathrm{Y}^{2}+\rho_{\star}+\mathrm{SPC}$ \\
\hline Age (Gyr) & $7.8 \pm 5.0$ & $\mathrm{Y}^{2}+\rho_{\star}+\mathrm{SPC}$ \\
\hline$A_{V}(\mathrm{mag})^{\mathrm{c}}$ & $0.186 \pm 0.058$ & $\mathrm{Y}^{2}+\rho_{\star}+\mathrm{SPC}$ \\
\hline Distance (pc) & $257_{-12}^{+15}$ & $\mathrm{Y}^{2}+\rho_{\star}+\mathrm{SPC}$ \\
\hline
\end{tabular}

Notes.

a SPC-"Stellar Parameter Classification" method based on cross-correlating high-resolution spectra against synthetic templates (Buchhave et al. 2012). These parameters rely primarily on SPC, but have a small dependence also on the iterative analysis incorporating the isochrone search and global modeling of the data, as described in the text.

${ }^{\mathrm{b}}$ Isochrones $+\rho_{\star}+\mathrm{SPC}=$ Based on the $\mathrm{Y}^{2}$ isochrones ( $\mathrm{Yi}$ et al. 2001), the stellar density used as a luminosity indicator, and the SPC results.

${ }^{\mathrm{c}}$ Total $V$ band extinction to the star determined by comparing the catalog broad-band photometry listed in the table to the expected magnitudes from the Isochrones $+\rho_{\star}+$ SPC model for the star. We use the Cardelli et al. (1989) extinction law.

et al. 2012). The resulting $T_{\text {eff }}$ and $[\mathrm{Fe} / \mathrm{H}]$ measurements were combined with the stellar density $\rho_{\star}$ determined through our joint light curve and RV curve analysis, to determine the stellar mass, radius, age, luminosity, and other physical parameters, by comparison with the Yonsei-Yale $\left(\mathrm{Y}^{2}\right.$; Yi et al. 2001) stellar evolution models (see Figure 4). This provided a revised estimate of $\log g_{\star}$ which was fixed in a second iteration of SPC. Our final adopted stellar parameters are listed in Table 4 . We find that the star HATS-7 has a mass of $0.849 \pm 0.027 M_{\odot}$, a radius of $0.815_{-0.035}^{+0.049} R_{\odot}$, and is at a reddening-corrected distance of $261_{-12}^{+16} \mathrm{pc}$. Note that the error on the distance does
Table 5

Parameters for the Transiting Planet HATS-7b

\begin{tabular}{|c|c|}
\hline Parameter & Value $^{\mathrm{a}}$ \\
\hline \multicolumn{2}{|l|}{ Light curve parameters } \\
\hline$P$ (days) & $3.1853150 \pm 0.0000054$ \\
\hline$T_{c}(\mathrm{BJD})^{\mathrm{b}}$ & $2456528.29697 \pm 0.00058$ \\
\hline$T_{14}$ (days) $^{\mathrm{b}}$ & $0.0958 \pm 0.0012$ \\
\hline 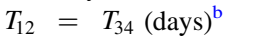 & $0.00735 \pm 0.00099$ \\
\hline$a / R_{\star}$ & $10.59_{-0.67}^{+0.51}$ \\
\hline$\zeta / R_{\star}^{\mathrm{c}}$ & $22.62 \pm 0.16$ \\
\hline$R_{\mathrm{p}} / R_{\star}$ & $0.0711 \pm 0.0019$ \\
\hline$b^{2}$ & $0.148_{-0.085}^{+0.101}$ \\
\hline$b \equiv a \cos i / R_{\star}$ & $0.39_{-0.13}^{+0.11}$ \\
\hline$i(\mathrm{deg})$ & $87.92 \pm 0.75$ \\
\hline \multicolumn{2}{|c|}{ Limb-darkening coefficients ${ }^{\mathrm{d}}$} \\
\hline$c_{1}, i$ (linear term) & 0.4140 \\
\hline$c_{2}, i$ (quadratic term) & 0.2464 \\
\hline$c_{1}, r$ & 0.5519 \\
\hline$c_{2}, r$ & 0.1984 \\
\hline \multicolumn{2}{|l|}{$\mathrm{RV}$ parameters } \\
\hline$K\left(\mathrm{~m} \mathrm{~s}^{-1}\right)$ & $18.4 \pm 1.9$ \\
\hline$e^{\mathrm{e}}$ & $<0.170$ \\
\hline $\mathrm{RV}$ jitter $\left(\mathrm{m} \mathrm{s}^{-1}\right)^{\mathrm{f}}$ & $3.0 \pm 1.1$ \\
\hline \multicolumn{2}{|l|}{ Planetary parameters } \\
\hline$M_{\mathrm{p}}\left(M_{\mathrm{J}}\right)$ & $0.120 \pm 0.012$ \\
\hline$R_{\mathrm{p}}\left(R_{\mathrm{J}}\right)$ & $0.563_{-0.034}^{+0.046}$ \\
\hline$C\left(M_{\mathrm{p}}, R_{\mathrm{p}}\right)^{\mathrm{g}}$ & -0.02 \\
\hline$\rho_{\mathrm{p}}\left(\mathrm{g} \mathrm{cm}^{-3}\right)$ & $0.83 \pm 0.18$ \\
\hline $\log g_{\mathrm{p}}(\mathrm{cgs})$ & $2.968 \pm 0.076$ \\
\hline$a(\mathrm{AU})$ & $0.04012 \pm 0.00043$ \\
\hline$T_{\mathrm{eq}}(\mathrm{K})^{\mathrm{h}}$ & $1084 \pm 32$ \\
\hline$\Theta^{i}$ & $0.0200 \pm 0.0024$ \\
\hline$\langle F\rangle\left(10^{9} \mathrm{erg} \mathrm{s}^{-1} \mathrm{~cm}^{-2}\right)^{\mathrm{i}}$ & $3.12_{-0.32}^{+0.45}$ \\
\hline
\end{tabular}

Notes.

${ }^{\mathrm{a}}$ The adopted parameters assume a circular orbit. Based on the Bayesian evidence ratio we find that this model is strongly preferred over a model in which the eccentricity is allowed to vary in the fit. For each parameter we give the median value and $68.3 \%(1 \sigma)$ confidence intervals from the posterior distribution.

${ }^{\mathrm{b}}$ Reported times are in Barycentric Julian Date calculated directly from UTC, without correction for leap seconds. $T_{c}$ : reference epoch of mid transit that minimizes the correlation with the orbital period. $T_{14}$ : total transit duration, time between first to last contact; $T_{12}=T_{34}$ : ingress/egress time, time between first and second, or third and fourth contact.

${ }^{\mathrm{c}}$ Reciprocal of the half duration of the transit used as a jump parameter in our MCMC analysis in place of $a / R_{\star}$. It is related to $a / R_{\star}$ by the expression $\zeta / R_{\star}=a / R_{\star}(2 \pi(1+e \sin \omega)) /\left(P \sqrt{1-b^{2}} \sqrt{1-e^{2}}\right)$ (Bakos et al. 2010). ${ }^{\mathrm{d}}$ Values for a quadratic law, adopted from the tabulations by Claret (2004) according to the spectroscopic (SPC) parameters listed in Table 4.

${ }^{\mathrm{e}}$ The $95 \%$ confidence upper-limit on the eccentricity from a model in which the eccentricity is allowed to vary in the fit.

${ }^{\mathrm{f}}$ Error term, either astrophysical or instrumental in origin, added in quadrature to the formal RV errors for the listed instrument. This term is varied in the fit assuming a prior inversely proportional to the jitter.

${ }^{\mathrm{g}}$ Correlation coefficient between the planetary mass $M_{\mathrm{p}}$ and radius $R_{\mathrm{p}}$ determined from the parameter posterior distribution via $C\left(M_{\mathrm{p}}, R_{\mathrm{p}}\right)=\left\langle\left(M_{\mathrm{p}}-\left\langle M_{\mathrm{p}}\right\rangle\right)\left(R_{\mathrm{p}}-\left\langle R_{\mathrm{p}}\right\rangle\right) /\left(\sigma_{M_{\mathrm{p}}} \sigma_{R_{\mathrm{p}}}\right)\right\rangle$, where $\langle\cdot\rangle$ is the expectation value operator, and $\sigma_{x}$ is the standard deviation of parameter $x$.

${ }^{\mathrm{h}}$ Planet equilibrium temperature averaged over the orbit, calculated assuming a Bond albedo of zero, and that flux is re-radiated from the full planet surface.

${ }^{\mathrm{i}}$ The Safronov number is given by $\Theta=\frac{1}{2}\left(V_{\text {esc }} / V_{\text {orb }}\right)^{2}=\left(a / R_{\mathrm{p}}\right)\left(M_{\mathrm{p}} / M_{\star}\right)$ (see Hansen \& Barman 2007).

${ }^{\mathrm{j}}$ Incoming flux per unit surface area, averaged over the orbit. 


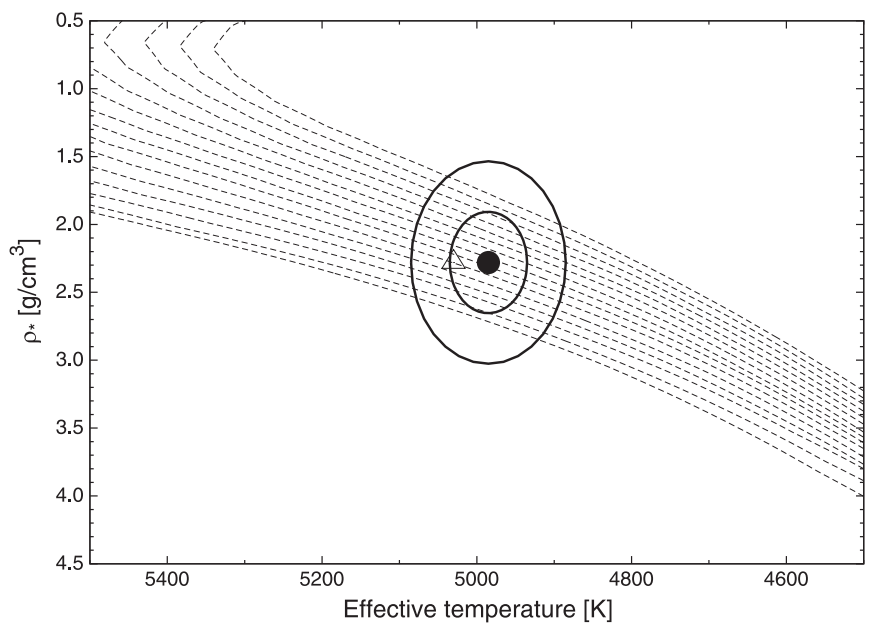

Figure 4. Comparison between the measured values of $T_{\mathrm{eff} \star}$ and $\rho_{\star}$ (from SPC applied to the HIRES $I_{2}$-free template spectrum, and from our modeling of the light curves and $\mathrm{RV}$ data, respectively), and the $\mathrm{Y}^{2}$ model isochrones from $\mathrm{Yi}$ et al. (2001). The best-fit values (dark filled circle), and approximate $1 \sigma$ and $2 \sigma$ confidence ellipsoids are shown. The values from our initial SPC iteration are shown with the open triangle. The $\mathrm{Y}^{2}$ isochrones are shown for ages of $0.2 \mathrm{Gyr}$, and 1.0-14.0 Gyr in $1 \mathrm{Gyr}$ increments.

not take into account the systematic errors due to uncertainties in the stellar isochrones. HATS-7 is a main-sequence star, and has a poorly constrained age due to the limited stellar evolution expected within the age of the universe at this stellar mass.

We also carried out a joint analysis of the HIRES RVs (fit using a Keplerian orbit) and the HATSouth, LCOGT $1 \mathrm{~m}$, and GROND light curves (fit using a Mandel \& Agol 2002 transit model with fixed quadratic limb darkening coefficients taken from Claret 2004) to measure the stellar density, as well as the orbital and planetary parameters. This analysis makes use of a differential evolution Markov Chain Monte Carlo procedure (ter Braak 2006) to estimate the posterior parameter distributions, which we use to determine the median parameter values and their $1 \sigma$ uncertainties. We also varied the jitter as a free parameter in the fit following Hartman et al. (2014), where we use the empirical distribution of the Keck/HIRES RV jitter values for low-activity cool main sequence stars from Wright (2005) to place a prior on the jitter. The results are listed in Table 5. We find that the planet HATS-7b has a mass of $0.120 \pm 0.012 M_{\mathrm{J}}$, and a radius of $0.563_{-0.034}^{+0.046} R_{\mathrm{J}}$. We also find that the observations are consistent with a circular orbit. When the eccentricity is allowed to vary in the fit, we find $e=0.065 \pm 0.053$ and a $95 \%$ confidence upper-limit of $e<0.170$. The parameters listed in Table 5 were determined assuming a fixed circular orbit.

In order to rule out the possibility that HATS-7 is a blended stellar eclipsing binary system, we carried out a blend analysis of the photometric data following Hartman et al. (2012). We find that a model consisting of single star with a transiting planet fits the data better than any of the blended stellar eclipsing binary models that we tested, but there are some blend models which cannot be rejected with greater than $5 \sigma$ confidence based on the photometry alone. In these models the primary star in the background eclipsing binary has a distance modulus that is no more than $2.1 \mathrm{mag}$ greater than that of a brighter foreground star. We simulated the CCFs, RVs, and BSs that would be measured with HIRES/Keck-I for these blend scenarios and found that in almost all cases the object would be easily identified as a composite stellar system (the CCFs would be double-peaked, or the BSs and/or RVs would vary by greater than $100 \mathrm{~m} \mathrm{~s}^{-1}$ ). We searched the spectrum for the spectral signature of a secondary star as described in Kolbl et al. (2015), and found no companions down to $1 \%$ of the brightness of the primary at separations in RV greater than $\pm 10 \mathrm{~km} \mathrm{~s}^{-1}$. There is a small region of parameter space where the BS and RV scatter for the blended system would be comparable to the measured scatters. These are blends where the background binary has a distance modulus that is between 1.95 and 2.1 mag greater than that of the foreground star, and where the RV of the foreground star differs by at least 50 $\mathrm{km} \mathrm{s}^{-1}$ from the systemic RV of the binary. Considering, however, that these blends can still be rejected with $4 \sigma$ confidence based on the photometry, and that the simulated $\mathrm{RV}$ s do not show the sinusoidal variation in orbital phase that is observed, we conclude that these blend scenarios do not provide a good description of the observations, and that HATS7 is a transiting planet system.

While we can rule out the possibility that HATS-7 is a blended stellar eclipsing binary system, we cannot rule out the possibility that HATS-7 is a transiting planet system with a fainter unresolved stellar companion. We note that this is often the case for many known transiting exoplanet systems. We simulated this scenario as well, and find that companions with masses up to that of the planet host cannot be ruled out. The highest spatial resolution images we have available are from the HIRES guide camera based on which we can rule out companions with $\Delta V<4$ at a separation greater than 1."5. High mass companions would also need to have been observed near conjunction for the system not to have been detected as a spectroscopic binary. Higher spatial resolution imaging and/or additional RV monitoring would be needed to put tighter constraints on any stellar companions. If HATS-7 does have a stellar companion, the radius and mass of the planet HATS-7b would be somewhat larger than what we infer here.

\section{DISCUSSION}

With its mass of $M_{\mathrm{p}}=0.120 \pm 0.012 M_{\mathrm{J}}$, HATS-7b is one of the very few transiting and well characterized superNeptunes known to date. We adopt a nomenclature, whereby planets with $0.18 M_{\mathrm{J}}<M_{\mathrm{p}} \leqslant 0.3 M_{\mathrm{J}}$, i.e., those more massive than halfway between Neptune and Saturn, are referred to as sub-Saturns (especially those toward the lower end of this mass range), whereas planets with $0.054 M_{\mathrm{J}}<M_{\mathrm{p}} \leqslant 0.18 M_{\mathrm{J}}$ as super-Neptunes (especially those toward the higher mass end). This nomenclature is more or less consistent with previous literature, e.g., HAT-P-18b (Hartman et al. 2011b) is a called a sub-Saturn $\left(M_{\mathrm{p}}=0.197 M_{\mathrm{J}}\right)$, while Kepler-101b (Bonomo et al. 2014) is called a super-Neptune $\left(M_{\mathrm{p}}=0.16 M_{\mathrm{J}}\right)$. The mass versus radius of low mass $\left(M_{\mathrm{p}}<0.2 M_{\mathrm{J}}\right)$ transiting planets with accurately determined masses (here we use $<20 \%$ uncertainty) is shown in Figure 5. It is noteworthy that only a handful of super-Neptunes are known (with accurate parameters), and the regime between $0.1 M_{\mathrm{J}}$ and $0.18 M_{\mathrm{J}}$ is occupied by only five such objects. These are Kepler-9c ( $K=12.34$ mag; Torres et al. 2011), Kepler-101b ( $K=12.0$; Bonomo et al. 2014), the recently discovered HATS-8b ( $K=12.6$; Bayliss et al. 2015), Kepler-35b $(K=13.9$; Welsh et al. 2012), and HATS-7b $(K=10.97)$, the subject of this paper. Among these planet hosting stars, HATS-7 is by far the brightest, which will greatly facilitate detailed studies. All low- 


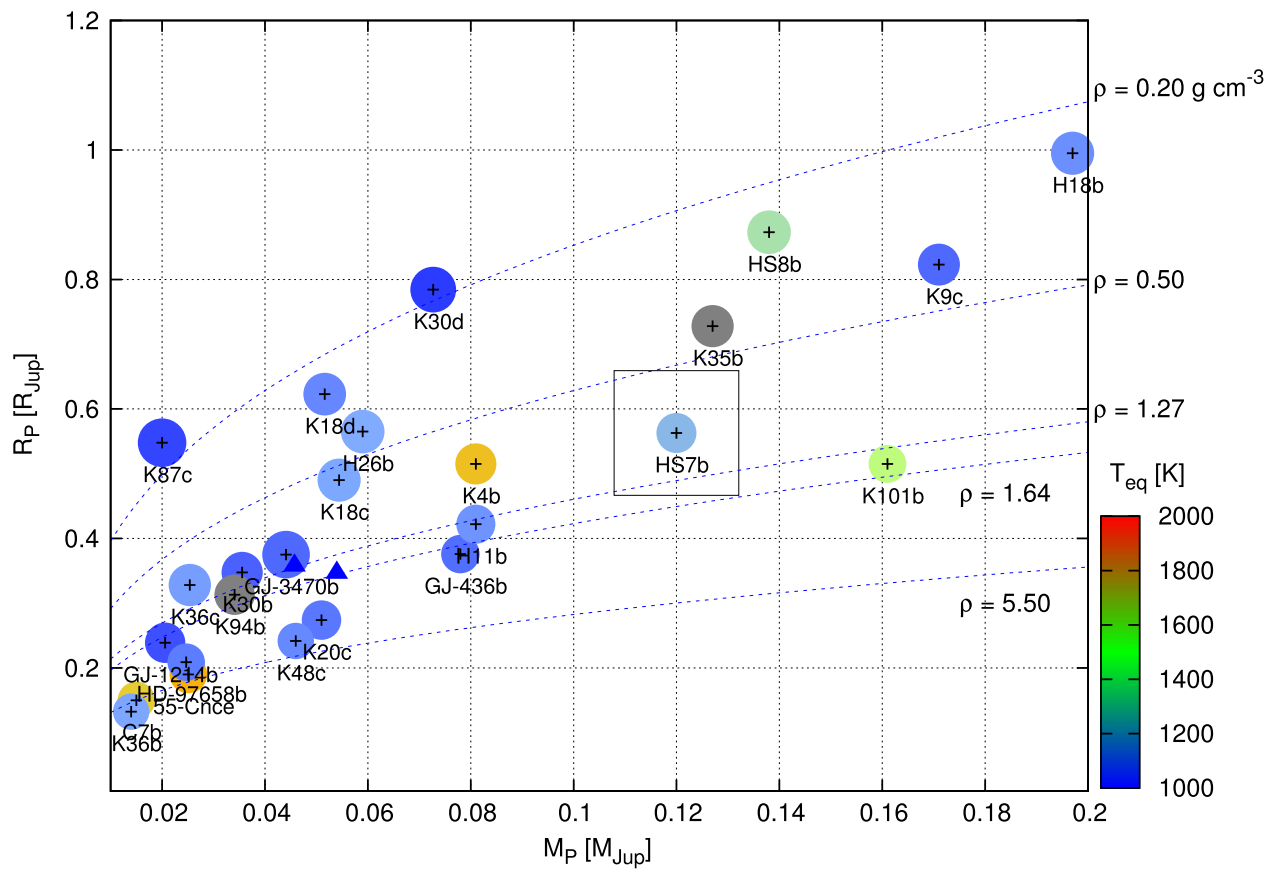

Figure 5. Mass-radius diagram of super-Neptunes $\left(M_{\mathrm{p}} \leqslant 0.18 M_{\mathrm{J}}\right)$ and super-Earths with accurately measured masses and radii $(<20 \%$ uncertainties). Color indicates equilibrium temperature (with the palette of $R, G, B=2000,1500,1000 \mathrm{~K}$ ), while size is inversely proportional to surface gravity. HATS-7b is also marked with a large box. Isodensity lines for $\rho=0.2,0.5,1.27$ (Uranus), 1.64 (Neptune), 5.5 (Earth) $\mathrm{g} \mathrm{cm}^{-3}$ are shown with dashed lines. Neptune is marked with a blue triangle. Abbreviations are: K: Kepler, H: HAT, HS: HATSouth, C: Corot.

mass transiting planets shown in Figure 5 were found by either Kepler and Corot (space-born surveys), HATNet and HATSouth (ground-based wide-field surveys), the pointed groundbased survey MEarth (GJ 1214b; Charbonneau et al. 2009; Irwin et al. 2009), or they were found by the photometric follow-up of planetary systems that were first discovered by RVs.

Well characterized planets and host stars in this regime are important in exploring the correlations between various system parameters. One example is the host star metallicity-planet occurrence rate correlation, which has been known for a long time (Fischer \& Valenti 2005) to exist for Jupiter size planets, and which correlation was found to be weakened for small, $<4 R_{\oplus}$ planets by Buchhave et al. (2012). However, recent analysis by Wang \& Fischer (2015) shows that the planet occurrence rate-metallicity correlation is universal, including for terrestrial planets. Using an increased sample and new analysis, Buchhave et al. (2014) find a correlation between host star metallicity and planet radius, in the sense that the average metallicity of the host star increases with planet size. These authors also find three populations of exoplanets (rocky, gasdwarf, giant), as based on the metallicity of the stars. The discovery of HATS-7b around a metal-rich $([\mathrm{Fe} / \mathrm{H}]$ $=+0.250 \pm 0.080)$ star, and the apparent lack of a hot Jupiter in this system will help in refining such future analyses.

The mass regime of super Neptunes is important in studying the transition from ice giants to gas giants, where, in the core accretion scenario (e.g., Mordasini et al. 2015), rapid accumulation of a gaseous envelope is expected to start. This transition is well demonstrated in Figure 6, which plots the bulk density of transiting exoplanets as a function of their masses (again, only for those with reliable parameters determined to better than $20 \%$ precision). Mean density first decreases with increasing planetary mass, as the planetary mass increases from
super-Earths $\left(\sim 0.01 M_{\mathrm{J}}\right)$ to Neptunes $\left(\sim 0.1 M_{\mathrm{J}}\right)$. Then, in the super-Neptune/sub-Saturn regime, the trend reverses, as matter becomes increasingly degenerate under high pressure, and density starts to increase.

With the increasing number of well characterized transiting exoplanets, we are gradually mapping out the parameter space these planets occupy. While it is customary to explore the relations between various parameters (e.g., mass, radius, equilibrium temperature), here we examine the boundaries spanned in the planetary mass-density domain. The approximate envelopes of the distribution are:

$$
M_{\mathrm{p}} \lesssim 0.02 M_{\mathrm{J}}:\left\{\begin{array}{l}
\rho_{\mathrm{p}} \gtrsim 14\left(M_{\mathrm{p}} / M_{\mathrm{J}}\right)^{0.178} \\
\rho_{\mathrm{p}} \lesssim 25\left(M_{\mathrm{p}} / M_{\mathrm{J}}\right)^{0.178},
\end{array}\right.
$$

i.e., for rocky planets without significant amount of volatiles,

$$
M_{\mathrm{p}} \lesssim 0.4 M_{\mathrm{J}}:\left\{\begin{aligned}
\rho_{\mathrm{p}} & \gtrsim 3 / 4 \times 10^{-4}\left(M_{\mathrm{p}} / M_{\mathrm{J}}\right)^{-2} \\
\rho_{\mathrm{p}} & \lesssim 3 / 5\left(M_{\mathrm{p}} / M_{\mathrm{J}}\right)^{-3 / 4},
\end{aligned}\right.
$$

i.e., for Neptunes and Saturns, and

$$
M_{\mathrm{p}} \gtrsim 0.4 M_{\mathrm{J}}:\left\{\begin{array}{l}
\rho_{\mathrm{p}} \gtrsim 1 / 5\left(M_{\mathrm{p}} / M_{\mathrm{J}}\right)^{3 / 2} \\
\rho_{\mathrm{p}} \lesssim 5 / 2\left(M_{\mathrm{p}} / M_{\mathrm{J}}\right)^{1.1}
\end{array}\right.
$$

for ice and gas giants ( $\rho_{\mathrm{p}}$ measured in $\mathrm{g} \mathrm{cm}^{-3}$ for all). Here the $\rho_{\mathrm{p}} \propto\left(M_{\mathrm{p}} / M_{\mathrm{J}}\right)^{0.178}$ relation for the smallest planets is taken from Sotin et al. (2007), and is based on models. The other relations are approximate, and are not physically motivated, though it is possible that physical explanations for them do exist. Between earths and super-earths, the mean density only increases slightly. Once $M_{\mathrm{p}} \gtrsim 0.02 M_{\mathrm{J}}\left(6 M_{\oplus}\right)$ is reached, the bulk 


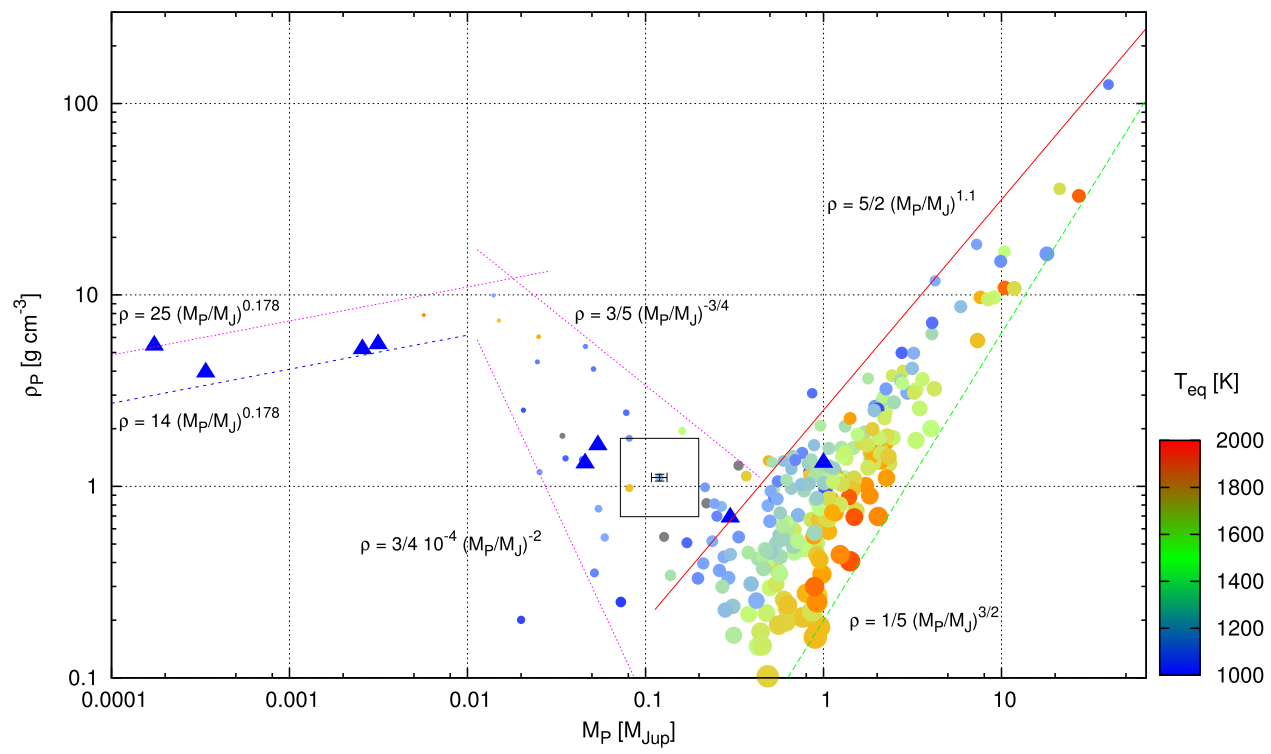

Figure 6. Planetary mean density as a function of planetary mass for planets with mass measured to better than $20 \%$ precision. HATS-7b is also marked with a large box. The size of the points scales with planetary radius, while the color indicates equilibrium temperature (with the palette of $R, G, B=2000,1500,1000 \mathrm{~K}$ ). It is clearly visible that the less dense Jupiter-mass planets are predominantly "hot" (red). The four thin lines indicate approximate boundaries of the current transiting extrasolar planet population in this space. The significant outlier on the bottom left side is Kepler-87c (Ofir et al. 2014), and the other significant outlier at $M_{\mathrm{p}} \approx 1 M_{\mathrm{J}}$ and $\rho \approx 3 \mathrm{~g} \mathrm{~cm}^{-3}$ is WASP-59b (Hébrard et al. 2013). Solar system planets are marked with blue triangles.

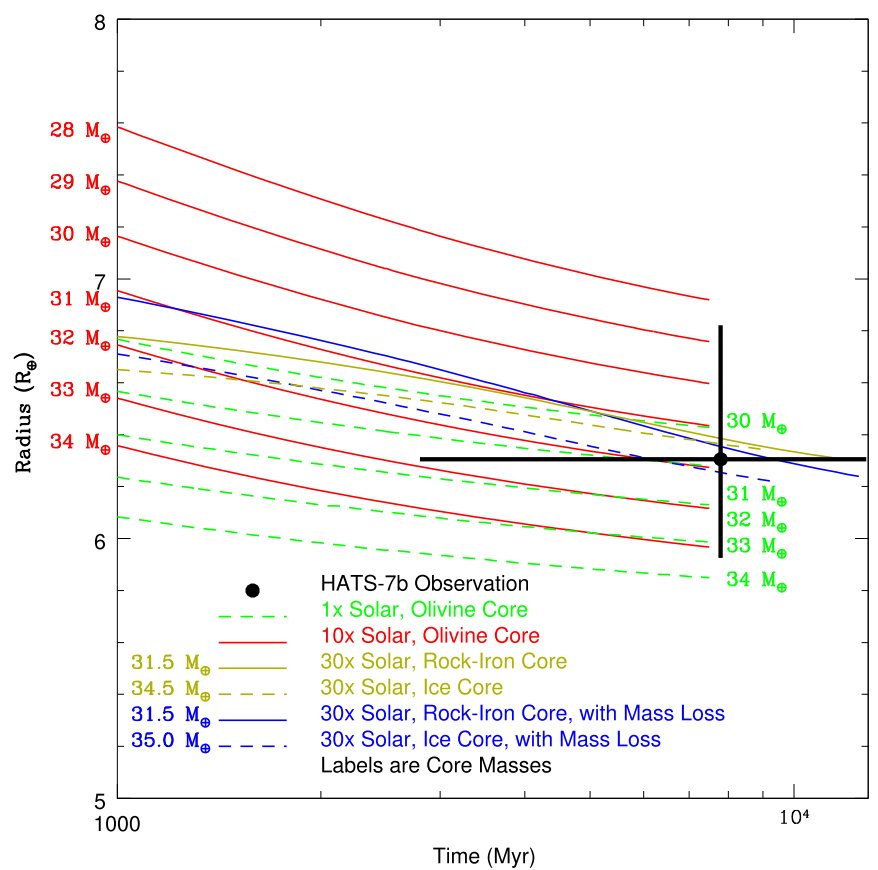

Figure 7. Radius vs. time for representative evolutionary models plotted against the observed radius and age estimates for HATS-7b. Models are labeled with their corresponding core masses. We include models with core masses from 30 to $34 M_{\oplus}$ with a $\mathrm{H}_{2}-\mathrm{He}$ atmosphere with solar metallicity, from 28 to $34 M_{\oplus}$ with an atmosphere with 10x solar metallicity, and four best-fit models with 30x solar metallicity. In all cases, we fit the models to a (final) total mass of $38 M_{\oplus}$.

density decreases as a result of retaining more volatiles, and the ability to form a more extended atmosphere. Finally, at around $M_{\mathrm{p}} \gtrsim 0.2 M_{\mathrm{J}} \quad\left(\sim 60 M_{\oplus}\right)$, the compression of matter gets significant to result in an increasing mean density.

The lower limit for the density of gaseous planets (dashed line in Figure $6, \propto M_{\mathrm{p}}^{3 / 2}$ ) is related to the inflation mechanism of planets, which has not been fully solved. It is noteworthy that this relation holds over two orders of magnitude in mass, from $M_{\mathrm{p}} \approx 0.5 M_{\mathrm{J}}$ to $50 M_{\mathrm{J}}$. The upper limit for the density of rocky planets (dotted line, $\propto M_{\mathrm{p}}^{-3 / 4}$ ) is likely to suffer from the bias against discovering compact rocky/iron planets, due to their smaller transit signatures. Also, while there are close to 250 well characterized transiting exoplanets, considering the dimensionality of the parameter space (e.g., $M_{\mathrm{p}}, R_{\mathrm{p}}, M_{\star}, P$, $[\mathrm{Fe} / \mathrm{H}], T_{\text {eff } \star}$, age), this is still a very small number to understand their distribution. Consequently, significant biases in the observed distribution exist. Probably the most important such bias is that the majority of the planets plotted in Figure 6 have short periods. The confirmation and characterization of long period planets takes much more time, and these systems are primarily discovered by Kepler, so the host stars are typically faint, further hindering follow-up studies.

The bulk composition of HATS-7b may be estimated by fitting theoretical structural and evolutionary models to the observed mass and radius of $38.0 \pm 3.9 M_{\oplus} \quad(0.120 \pm$ $\left.0.012 M_{\mathrm{J}}\right)$ and $6.31_{-0.38}^{+0.52} R_{\oplus}\left(0.563_{-0.034}^{+0.046} R_{\mathrm{J}}\right)$. We compute a range of models for planets of HATS-7b's mass for various possible parameters using the methodology of Howe \& Burrows (2015). If a two-layer partitioning with a rock-iron core and a hydrogen-helium envelope is assumed, the best fit models suggest a core mass of $31 \pm 4 M_{\oplus}$ and an envelope mass of $7 \pm 1.5 M_{\oplus}$, that is, a hydrogen-helium fraction of 18 $\pm 4 \%$. This result is consistent within uncertainty across a range of metallicity and whether or not evaporative mass loss is incorporated in the model. More quantitatively, increasing the metallicity from $1 \times$ solar to $10 \times$ solar increases the radius at $7.8 \mathrm{Gyr}$ by about $0.2 R_{\oplus}\left(0.018 R_{\mathrm{J}}\right)$, approximately the same amount as decreasing the core mass by $1 M_{\oplus}$. This is also of the same order as the uncertainty introduced by the uncertainty in age. Models with significantly smaller cores, $\lesssim 25 M_{\oplus}$, are ruled out because they under no circumstances shrink to the observed radius of the planet over the age of the system. For comparison, final radii for the $10 \times$ solar models with core 


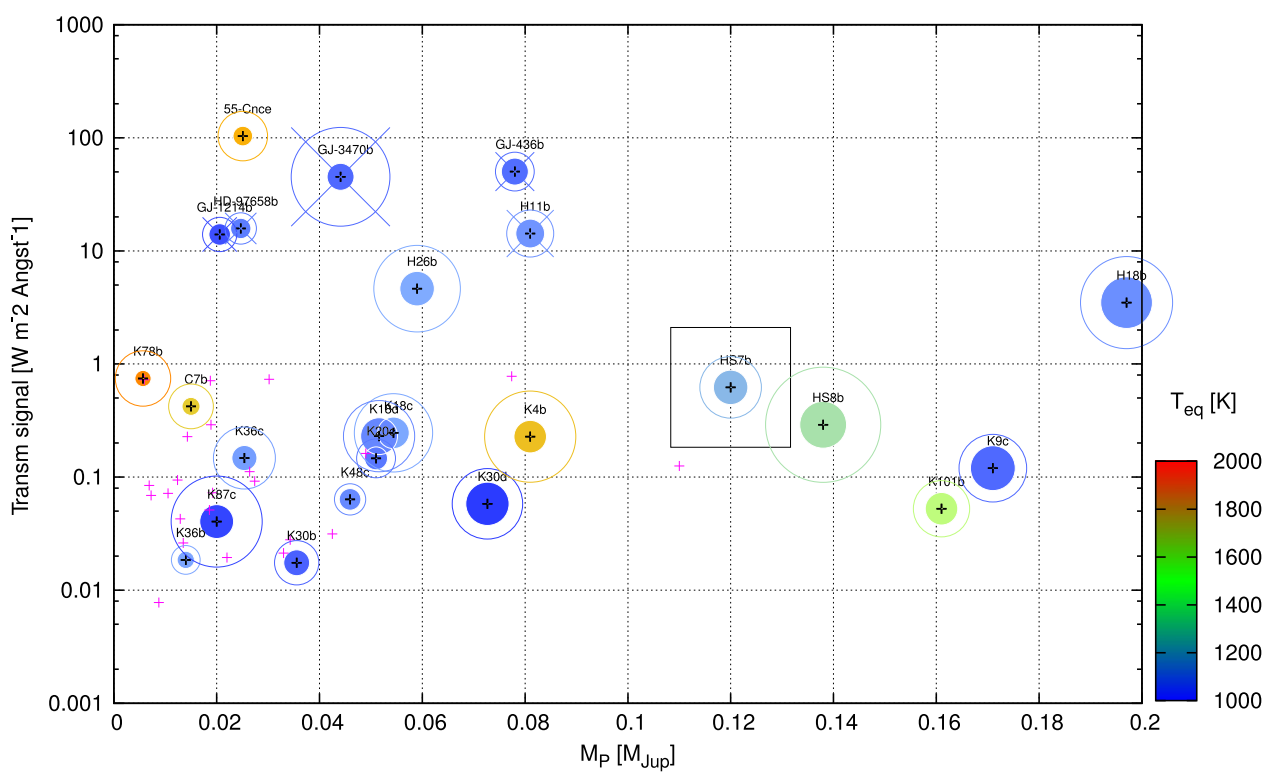

Figure 8. Approximate detectability of a planet's atmosphere in transmission as a function of planetary mass for super-Neptunes $\left(M_{\mathrm{p}} \leqslant 0.18 M_{\mathrm{J}}\right)$ and super-Earths. In calculating the scale height of the planet atmosphere we assume the molecular weight of pure molecular hydrogen, and the equilibrium temperature and surface gravity of the planet determined as from our analysis (Table 5). The fractional contribution of the transmission signal to the star light is approximated as $\delta=5 \times 2 R_{\mathrm{p}} H / R_{\star}^{2}$ (Perryman 2014). We then calculate the $K$-band flux of the star, and multiply with $\delta$, to come up with an approximate measure of the transmission signal. We stress that this quantity does not take into account the detailed (expected) spectrum of the planetary atmosphere, but is an order of magnitude estimate of the signature. The size of the filled circles scales with the radius of the planet (arbitrary scale), and the radius of the open circles scales with the scale height of the atmosphere. Small plus symbols denote planets with uncertain mass or radius measurements (error >20\%). Abbreviations are: K: Kepler, H: HAT, HS: HATSouth, C: Corot.

masses of $0,5,10,15$, and $20 M_{\oplus}$ are $0.95,0.87,0.79,0.71$, and $0.65 R_{\mathrm{J}}$, respectively (all larger than the observed radius of $0.56 R_{\mathrm{J}}$ ), while a bare core of $38 M_{\oplus}$ has a radius of $0.202 R_{\mathrm{J}}$ if composed of rock-iron and $0.296 R_{\mathrm{J}}$ if composed of ice. In the case of a pure ice core, the best fit is a core mass of $34.5 \pm 4$ $M_{\oplus}$ and an envelope mass of $3.5 \pm 1.5 M_{\oplus}$, that is, a hydrogenhelium fraction of $9 \pm 4 \%$, similar to the composition of Uranus and Neptune (Helled et al. 2011). Figure 7 shows radius versus time for representative evolutionary models with core masses of 28-34 $M_{\oplus}$, encompassing the full range of core masses consistent with observations.

While the mean density of exoplanets is an important constraint on their bulk composition, detailed studies of the planetary atmospheres can provide a direct measurement of the composition of the planet. One important way to detect such compositions is via transmission spectroscopy. In Figure 8 we show super-Neptunes to super-Earths, including the sub-Saturn HAT-P-18b, with the expected transmission signatures plotted against the planetary masses. To derive an approximate measure of the transmission signatures, we approximated the $H$ scale height as $H=k_{\mathrm{B}} T_{\mathrm{eq}} /\left(\mu g_{\mathrm{p}}\right)$, where $k_{\mathrm{B}}$ is the Boltzmann constant, $T_{\mathrm{eq}}$ is the equilibrium temperature of the planets (assuming black bodies, and full redistribution of heat), $\mu$ is the mean molecular weight, and $g_{\mathrm{p}}$ is the planetary surface gravity. To make the comparison simpler (and because of lacking further information on their atmospheric composition), we used $\mu=2$ (i.e., $\mathrm{H}_{2}$ ) for all planets, even though the mean molecular weight of the atmospheres will be larger, and thus our estimates are somewhat optimistic. We then approximated the fractional contribution of transmission through the planet's atmosphere to the total stellar flux as $\delta=5 \times 2 R_{\mathrm{p}} H / R_{\star}^{2}$ (Perryman 2014), and multiplied this with the total estimated $K$-band flux of the star. Atmospheric transmission analysis has been carried out for five of these planets, typically those in the top left side of Figure 8 (those with low mass and high expected transmission signal). These are, in order of increasing planetary mass, GJ-1214b (Kreidberg et al. 2014), HD 97658b (Knutson et al. 2014b), GJ 3470b (Ehrenreich et al. 2014), GJ 436b (Knutson et al. 2014a), and HAT-P-11b (Fraine et al. 2014). With the exception of HAT-P$11 \mathrm{~b}$, all showed essentially featureless transmission spectra, indicating hazes, clouds, or atmospheres with high molecular weight. The only Neptune mass planet with features in its transmission spectrum is HAT-P-11b, where the signature of water was detected (Fraine et al. 2014). Among the super Neptunes, HATS-7b is the most promising target for detecting its atmosphere. Also, such measurements will be facilitated by the quiet host star; high-resolution spectroscopy of HATS-7 shows very low RV jitter, low $v \sin i$, and no chromospheric activity. Altogether, it is scientifically compelling to pursue transmission spectroscopy for the recently discovered super Neptunes HATS-7b and HATS- $8 b$, and the sub-Saturn HAT-P$18 \mathrm{~b}$, where relatively large signals are expected. Perhaps a trend from featureless spectra to feature-rich transmission (observed for certain hot Jupiters) will be observed with increasing planetary mass through the super-Neptune regime.

Development of the HATSouth project was funded by NSF MRI grant NSF/AST-0723074, operations have been supported by NASA grants NNX09AB29G and NNX12AH91H, and follow-up observations received partial support from grant NSF/AST-1108686. A.J. acknowledges support from FONDECYT project 1130857, BASAL CATA PFB-06, and project IC120009 "Millennium Institute of Astrophysics (MAS)" of the Millenium Science Initiative, Chilean Ministry of Economy. R.B. and N.E. are supported by CONICYT-PCHA/ Doctorado Nacional. R.B. and N.E. acknowledge additional support from project IC120009 "Millenium Institute of 
Astrophysics (MAS)" of the Millennium Science Initiative, Chilean Ministry of Economy. V.S. acknowledges support form BASAL CATA PFB-06. This work is based on observations made with ESO Telescopes at the La Silla Observatory. This paper also uses observations obtained with facilities of the Las Cumbres Observatory Global Telescope. Work at the Australian National University is supported by ARC Laureate Fellowship Grant FL0992131. We acknowledge the use of the AAVSO Photometric All-Sky Survey (APASS), funded by the Robert Martin Ayers Sciences Fund, and the SIMBAD database, operated at CDS, Strasbourg, France. Operations at the MPG $2.2 \mathrm{~m}$ Telescope are jointly performed by the Max Planck Gesellschaft and the European Southern Observatory. The imaging system GROND has been built by the high-energy group of MPE in collaboration with the LSW Tautenburg and ESO. The authors wish to recognize and acknowledge the very significant cultural role and reverence that the summit of Mauna Kea has always had within the indigenous Hawaiian community. We are most fortunate to have the opportunity to conduct observations from this mountain. G. Á. B. wishes to thank the warm hospitality of Adèle and Joachim Cranz at the farm Isabis, supporting the operations and service missions of HATSouth.

\section{REFERENCES}

Bakos, G. Á., Csubry, Z., Penev, K., et al. 2013, PASP, 125, 154 Bakos, G. Á., Torres, G., Pál, A., et al. 2010, ApJ, 710, 1724 Bayliss, D., Hartman, J. D., Bakos, G. Á., et al. 2015, arXiv:1506.01334 Bayliss, D., Zhou, G., Penev, K., et al. 2013, AJ, 146, 113 Bonfils, X., Gillon, M., Udry, S., et al. 2012, A\&A, 546, A27 Bonomo, A. S., Sozzetti, A., Lovis, C., et al. 2014, A\&A, 572, A2 Borucki, W. J., Koch, D., Basri, G., et al. 2010, Sci, 327, 977 Brown, T. M., Baliber, N., Bianco, F. B., et al. 2013, PASP, 125, 1031 Buchhave, L. A., Bizzarro, M., Latham, D. W., et al. 2014, Natur, 509, 593 Buchhave, L. A., Latham, D. W., Johansen, A., et al. 2012, Natur, 486, 375 Butler, R. P., Marcy, G. W., Williams, E., et al. 1996, PASP, 108, 500 Cardelli, J. A., Clayton, G. C., \& Mathis, J. S. 1989, ApJ, 345, 245 Charbonneau, D., Berta, Z. K., Irwin, J., et al. 2009, Natur, 462, 891 Claret, A. 2004, A\&A, 428, 1001

Dopita, M., Hart, J., McGregor, P., et al. 2007, Ap\&SS, 310, 255
Ehrenreich, D., Bonfils, X., Lovis, C., et al. 2014, A\&A, 570, A89 Fischer, D. A., \& Valenti, J. 2005, ApJ, 622, 1102

Fraine, J., Deming, D., Benneke, B., et al. 2014, Natur, 513, 526 Gillon, M., Pont, F., Demory, B.-O., et al. 2007, A\&A, 472, L13

Greiner, J., Bornemann, W., Clemens, C., et al. 2008, PASP, 120, 405

Hansen, B. M. S., \& Barman, T. 2007, ApJ, 671, 861

Hartman, J. D., Bakos, G. Á., Béky, B., et al. 2012, AJ, 144, 139

Hartman, J. D., Bakos, G. Á., Kipping, D. M., et al. 2011a, ApJ, 728, 138

Hartman, J. D., Bakos, G. Á., Sato, B., et al. 2011b, ApJ, 726, 52

Hartman, J. D., Bakos, G. Á., Torres, G., et al. 2014, AJ, 147, 128

Hébrard, G., Collier Cameron, A., Brown, D. J. A., et al. 2013, A\&A, 549, A134

Helled, R., Anderson, J. D., Podolak, M., \& Schubert, G. 2011, ApJ, 726, 15

Howard, A. W., Johnson, J. A., Marcy, G. W., et al. 2010, ApJ, 721, 1467

Howe, A. R., \& Burrows, A. S. 2015, ApJ, 808, 150

Irwin, J., Charbonneau, D., Nutzman, P., \& Falco, E. 2009, in IAU Symp. 253 Transiting Planets, ed. F. Pont, D. Sasselov \& M. J. Holman (Cambridge: Cambridge Univ. Press), 37

Jordán, A., Brahm, R., Bakos, G. Á., et al. 2014, AJ, 148, 29

Kaufer, A., \& Pasquini, L. 1998, Proc. SPIE, 3355, 844

Knutson, H. A., Benneke, B., Deming, D., \& Homeier, D. 2014a, Natur, 505,66

Knutson, H. A., Dragomir, D., Kreidberg, L., et al. 2014b, ApJ, 794, 155

Kolbl, R., Marcy, G. W., Isaacson, H., \& Howard, A. W. 2015, AJ, 149, 18

Kovács, G., Bakos, G., \& Noyes, R. W. 2005, MNRAS, 356, 557

Kovács, G., Zucker, S., \& Mazeh, T. 2002, A\&A, 391, 369

Kreidberg, L., Bean, J. L., Désert, J.-M., et al. 2014, Natur, 505, 69

Mandel, K., \& Agol, E. 2002, ApJL, 580, L171

Mohler-Fischer, M., Mancini, L., Hartman, J. D., et al. 2013, A\&A, 558, A55

Mordasini, C., Mollière, P., Dittkrist, K.-M., Jin, S., \& Alibert, Y. 2015, IJAsB, 14,201

Ofir, A., Dreizler, S., Zechmeister, M., \& Husser, T.-O. 2014, A\&A, 561, A103

Penev, K., Bakos, G. Á., Bayliss, D., et al. 2013, AJ, 145, 5

Perryman, M. 2014, The Exoplanet Handbook (Cambridge: Cambridge Univ. Press)

Queloz, D., Mayor, M., Udry, S., et al. 2001, Msngr, 105, 1

Sotin, C., Grasset, O., \& Mocquet, A. 2007, Icar, 191, 337

ter Braak, C. J. F. 2006, Statistics and Computing, 16, 239

Torres, G., Bakos, G. Á., Kovács, G., et al. 2007, ApJL, 666, L121

Torres, G., Fressin, F., Batalha, N. M., et al. 2011, ApJ, 727, 24

Vogt, S. S., Allen, S. L., Bigelow, B. C., et al. 1994, Proc. SPIE, 2198, 362

Wang, J., \& Fischer, D. A. 2015, AJ, 149, 14

Welsh, W. F., Orosz, J. A., Carter, J. A., et al. 2012, Natur, 481, 475

Wright, J. T. 2005, PASP, 117, 657

Yi, S., Demarque, P., Kim, Y.-C., et al. 2001, ApJS, 136, 417

Zhou, G., Bayliss, D., Hartman, J. D., et al. 2014, MNRAS, 437, 2831 